\title{
NEAR MDS POSET CODES AND DISTRIBUTIONS
}

\author{
ALEXANDER BARG AND PUNARBASU PURKAYASTHA
}

\begin{abstract}
We study $q$-ary codes with distance defined by a partial order of the coordinates of the codewords. Maximum Distance Separable (MDS) codes in the poset metric have been studied in a number of earlier works. We consider codes that are close to MDS codes by the value of their minimum distance. For such codes, we determine their weight distribution, and in the particular case of the "ordered metric" characterize distributions of points in the unit cube defined by the codes. We also give some constructions of codes in the ordered Hamming space.
\end{abstract}

\section{INTRODUCTION}

A set of points $C=\left\{c_{1}, \ldots, c_{M}\right\}$ in the $q$-ary $n$-dimensional Hamming space $\mathbb{F}_{q}^{n}$ is called a Maximum Distance Separable (MDS) code if the Hamming distance between any two distinct points of $C$ satisfies $d\left(c_{i}, c_{j}\right) \geq d$ and the number of points is $M=q^{n-d+1}$. By the well-known Singleton bound of coding theory, this is the maximum possible number of points with the given separation. If $C$ is an MDS code that forms an $\mathbb{F}_{q}$-linear space, then its dimension $k$, distance $d$ and length $n$ satisfy the relation $d=n-k+1$. MDS codes are known to be linked to classical old problems in finite geometry and to a number of other combinatorial questions related to the Hamming space $[19,1]$. At the same time, the length of MDS codes cannot be very large; in particular, in all the known cases, $n \leq q+2$. This restriction has led to the study of classes of codes with distance properties close to MDS codes, such as $t$-th rank MDS codes [22], near MDS codes [6] and almost MDS codes [5]. The distance of these codes is only slightly less than $n-k+1$, and at the same time they still have many of the structural properties associated with MDS codes.

In this paper we extend the study of linear near MDS (NMDS) codes to the case of the ordered Hamming space and more generally, to poset metrics. The ordered Hamming weight was introduced by Niederreiter [16] for the purpose of studying uniform distributions of points in the unit cube. The ordered Hamming space in the context of coding theory was first considered by Rosenbloom and Tsfasman [18] for a study of one generalization of Reed-Solomon codes (the ordered distance is therefore sometimes called the NRT distance). The ordered Hamming space and the NRT metric have multiple applications in coding theory including a generalization of the Fourier transform over finite fields [10, 14], list decoding of algebraic codes [17], and coding for a fading channel of special structure [18, 9]. This space also gives rise to a range of combinatorial problems. In the context of algebraic combinatorics, it

1991 Mathematics Subject Classification. Primary 94B25.

Key words and phrases. Poset metrics, ordered Hamming space, MDS codes.

This research supported in part by NSF grants DMS0807411, CCF0916919, CCF0830699, and CCF0635271. 
supports a formally self-dual association scheme whose eigenvalues form a family of multivariate discrete orthogonal polynomials $[13,3,2]$.

A particular class of distributions in the unit cube $U^{n}=[0,1)^{n}$, called $(t, m, n)$ nets, defined by Niederreiter in the course of his studies, presently forms the subject of a large body of literature. MDS codes in the ordered Hamming space and their relations to distributions and $(t, m, n)$-nets have been extensively studied $[18,20$, 7,11 . The ordered Hamming space was further generalized by Brualdi et al. in [4] which introduced metrics on strings defined by arbitrary partially ordered sets, calling them poset metrics.

The relation between MDS and NMDS codes in the ordered metric and distributions is the main motivation of the present study. As was observed by Skriganov [20], MDS codes correspond to optimal uniform distributions of points in the unit cube. The notion of uniformity is rather intuitive: an allocation of $M$ points forms a uniform distribution if every measurable subset $A \subset U^{n}$ contains a $\operatorname{vol}(A)$ proportion of the $M$ points (in distributions that arise from codes, this property is approximated by requiring that it hold only for some fixed collection of subsets). Skriganov [20] observes that distributions that arise from MDS codes are optimal in some well-defined sense. In the same way, NMDS codes correspond to distributions that are not far from optimal (they are characterized exactly below). Although the primary motivation is to study NMDS codes in the ordered metric, the calculations are easily generalized to the poset metric. We will hence derive the results in the general case of the poset metric, and mention the results in the ordered metric as specific cases.

The rest of the article is organized as follows. In the next section we provide basic definitions and some properties of near-MDS codes. We will also have a chance to discuss generalized Hamming weights of Wei [22] in the poset metric case. In Section 3 we show a relationship between distribution of points in the unit cube and NMDS codes. In Section 4 we determine the weight distribution of NMDS codes, and finally in Section 5, we provide some constructions of NMDS codes in the ordered Hamming space.

\section{DEFINITIONS AND BASIC PROPERTIES}

2-A. Poset metrics. We begin with defining poset metrics on $q$-ary strings of a fixed length and introduce the ordered Hamming metric as a special case of the general definition. Entries of a string $x=\left(x_{1}, x_{2}, \ldots\right)$ are indexed by a finite set $N$ which we call the set of coordinates. Let $\overrightarrow{\mathcal{P}}$ be an arbitrary partial order $(\leq)$ on $N$. Together $N$ and $\overrightarrow{\mathcal{P}}$ form a poset. An ideal of the poset is a subset $I \subset N$ that is "downward closed" under the $\leq$ relation, which means that the conditions $i, j \in N, j \in I$ and $i \leq j$ imply that $i \in I$. For the reasons that will become clear below, such ideals will be called left-adjusted (l.a.).

A chain is a linearly ordered subset of the poset. The dual poset $\overleftarrow{\mathcal{P}}$ is the set $N$ with the same set of chains as $\overrightarrow{\mathcal{P}}$, but the order within each of them reversed. In other words $j \leq i$ in $\overleftarrow{\mathcal{P}}$ if and only if $i \leq j$ in $\overrightarrow{\mathcal{P}}$. An ideal in the dual poset will be termed right-adjusted (r.a.). For a subset $S \subseteq \overrightarrow{\mathcal{P}}$ we denote by $\langle S\rangle=\langle S\rangle_{\overrightarrow{\mathcal{P}}}$ the smallest $\overrightarrow{\mathcal{P}}$-ideal containing the set $S$ (we write $S \subseteq \overrightarrow{\mathcal{P}}$ to refer to a subset $S \subseteq N$ whose elements are ordered according to $\overrightarrow{\mathcal{P}}$ ). The support of a sequence $x$ is the subset $\operatorname{supp} x \subseteq N$ formed by the indices of all the nonzero entries of $x$. The 
set $\langle\operatorname{supp} x\rangle \subseteq \overrightarrow{\mathcal{P}}$ will be called the l.a. support of $x$. The r.a. support is defined analogously.

Definition 2.1. (Brualdi et al. [4]) Let $\overrightarrow{\mathcal{P}}$ be a poset defined on $N$ and let $x, y \in$ $\mathbb{F}_{q}^{|N|}$ be two strings. Define the weight of $x$ with respect to $\overrightarrow{\mathcal{P}}$ as w $(x)=|\langle\operatorname{supp} x\rangle|$, i.e., the size of the smallest $\overrightarrow{\mathcal{P}}$-ideal that contains the support of $x$. The distance between $x$ and $y$ is defined as $d_{\overrightarrow{\mathcal{p}}}(x, y)=\mathrm{w}(x-y)=|\langle\operatorname{supp}(x-y)\rangle|$.

A code $\mathcal{C}$ of minimum distance $d$ is a subset of $\mathbb{F}_{q}^{|N|}$ such that any two distinct vectors $x$ and $y$ of $\mathcal{C}$ satisfy $d_{\overrightarrow{\mathcal{p}}}(x, y) \geq d$. It is similarly possible to consider codes whose distance is measured relative to $\overleftarrow{\mathcal{P}}$. In this paper we will be concerned with linear codes over a finite field by which we mean linear subspaces of $\mathbb{F}_{q}^{|N|}$. Given a linear code $\mathcal{C} \subset \mathbb{F}_{q}^{|N|}$ its dual code $\mathcal{C}^{\perp}$ is the set of vectors $\left\{y \in \mathbb{F}_{q}^{|N|}: \forall_{x \in \mathcal{C}} \sum_{i} x_{i} y_{i}=\right.$ $0\}$. The weights in the dual code $\mathcal{C}^{\perp}$ are considered with respect to the dual poset $\overleftarrow{\mathcal{P}}$

A subset of $\mathbb{F}_{q}^{|N|}$ is called an orthogonal array of strength $t$ and index $\theta$ with respect to $\overrightarrow{\mathcal{P}}$ if any $t$ l.a. columns contain any vector $z \in \mathbb{F}_{q}^{t}$ exactly $\theta$ times. In particular, the dual of a linear poset code is also a linear orthogonal array.

For instance, the Hamming metric is defined by the partial order $\overrightarrow{\mathcal{P}}$ which is a single antichain of length $n=|N|$ (no two elements are comparable). Accordingly, the distance between two sequences is given by the number of coordinates in which they differ. In this case, $\overrightarrow{\mathcal{P}}=\overleftarrow{\mathcal{P}}$

2-B. Ordered Hamming metric. The ordered Hamming metric is defined by a poset $\overrightarrow{\mathcal{P}}$ which is a disjoint union of $n$ chains of equal length $r$. Since we work with this metric in later sections of the paper, let us discuss it in more detail. In this case $N$ is a union of $n$ blocks of length $r$, and it is convenient to write a vector (sequence) as $x=\left(x_{11}, \ldots, x_{1 r}, \ldots, x_{n 1}, \ldots, x_{n r}\right) \in \mathbb{F}_{q}^{r, n}$. According to Definition 2.1 , the weight of $x$ is given by

$$
\mathrm{w}(x)=\sum_{i=1}^{n} \max \left(j: x_{i j} \neq 0\right) .
$$

For a given vector $x$ let $e_{i}, i=1, \ldots, r$ be the number of $r$-blocks of $x$ whose rightmost nonzero entry is in the $i$ th position counting from the beginning of the block. The $r$-vector $e=\left(e_{1}, \ldots, e_{r}\right)$ will be called the shape of $x$. For brevity we will write

$$
|e|=\sum_{i} e_{i}, \quad|e|^{\prime}=\sum_{i} i e_{i}, \quad e_{0}=n-|e| .
$$

For $I=\langle\operatorname{supp} x\rangle$ we will denote the shape of the ideal $I$ as shape $(I)=e$. By analogy with the properties of ideals in the ordered Hamming space, we use the term "left adjusted" for ideals in general posets $\overrightarrow{\mathcal{P}}$.

An $(n r, M, d)$ ordered code $\mathcal{C} \subset \mathbb{F}_{q}^{r, n}$ is an arbitrary subset of $M$ vectors in $\mathbb{F}_{q}^{r, n}$ such that the ordered distance between any two distinct vectors in $\mathcal{C}$ is at least $d$. If $\mathcal{C}$ is a linear code of dimension $k$ over $\mathbb{F}_{q}$ and minimum ordered distance $d$, we will denote it as an $[n r, k, d]$ code. The dual of $\mathcal{C}$, denoted as $\mathcal{C}^{\perp}$, is defined as $\mathrm{e}^{\perp}=\left\{x \in \mathbb{F}_{q}^{r, n}: \forall_{c \in \mathcal{C}} \sum_{i, j} x_{i j} c_{i j}=0\right\}$. The distance in $\mathrm{C}^{\perp}$ is derived from the dual order $\overleftarrow{\mathcal{P}}$, i.e., from the r.a. ideals. 
The notion of orthogonal arrays in the ordered Hamming space is derived from the general definition. They will be called ordered orthogonal arrays (OOAs) below. We write $(t, n, r, q)$ OOA for an orthogonal array of strength $t$ in $\mathbb{F}_{q}^{r, n}$. Combinatorics of the ordered Hamming space and the duality between codes and OOAs was studied in detail by Martin and Stinson [13], Skriganov [20], and the present authors [2].

2-C. NMDS poset codes. We begin our study of NMDS codes in the poset space with several definitions that are generalized directly from the corresponding definitions in the Hamming space $[22,6]$. The $t$-th generalized poset weight of a linear $[n, k]$ code $\mathcal{C}$ is defined as

$$
d_{t}(\mathcal{C}) \triangleq \min \{|\langle\operatorname{supp} \mathcal{D}\rangle|: \mathcal{D} \text { is an }[n, t] \text { subcode of } \mathcal{C}\} \text {, }
$$

where supp $\mathcal{D}$ is the union of the supports of all the vectors in $\mathcal{D}$. Note that $d_{1}(\mathcal{C})=d$, the minimum distance of the code $\mathcal{C}$. Generalized poset weights have properties analogous to the well-known set of properties of generalized Hamming weights.

Lemma 2.2. Let $\mathcal{C}$ be a linear $[n, k]$ poset code in $\mathbb{F}_{q}^{n}$. Then

(1) $0<d_{1}(\mathrm{C})<d_{2}(\mathrm{C})<\cdots<d_{k}(\mathrm{C}) \leq n$.

(2) Generalized Singleton bound: $d_{t}(\overline{\mathcal{C}}) \leq n-\operatorname{dim}(\mathcal{C})+t, \quad \forall t \geq 1$.

(3) If $\mathrm{C}^{\perp}$ is the dual code of $\mathrm{C}$ then

$\left\{d_{1}(\mathcal{C}), d_{2}(\mathcal{C}), \ldots, d_{k}(\mathcal{C})\right\} \cup\left(n+1-\left\{d_{1}\left(\mathrm{C}^{\perp}\right), d_{2}\left(\mathrm{C}^{\perp}\right), \ldots, d_{n-k}\left(\mathrm{C}^{\perp}\right)\right\}\right)=\{1, \ldots, n\}$.

(4) Let $H$ be the parity check matrix of $\mathcal{C}$. Then $d_{t}(\mathrm{C})=\delta$ if and only if

(a) Every $\delta-1$ l.a. columns of $H$ have rank at least $\delta-t$.

(b) There exist $\delta$ l.a. columns of $H$ with rank exactly $\delta-t$.

Proof. (1) Let $\mathcal{D}_{t} \subseteq \mathcal{C}$ be a linear subspace such that $\left|\left\langle\operatorname{supp} \mathcal{D}_{t}\right\rangle\right|=d_{t}(\mathcal{C})$ and $\operatorname{rank}\left(\mathcal{D}_{t}\right)=t, t \geq 1$. Let $\Omega\left(\mathcal{D}_{t}\right)$ denote the maximal elements of the ideal $\left\langle\operatorname{supp} \mathcal{D}_{t}\right\rangle$. For each coordinate in $\Omega\left(\mathcal{D}_{t}\right), \mathcal{D}_{t}$ has at least one vector with a nonzero component in that coordinate. We pick $i \in \Omega\left(\mathcal{D}_{t}\right)$ and let $\mathcal{D}_{t}^{i}$ be obtained by retaining only those vectors $v$ in $\mathcal{D}_{t}$ which have $v_{i}=0$. Then

$$
d_{t-1}(\mathcal{C}) \leq\left|\left\langle\operatorname{supp} \mathcal{D}_{t}^{i}\right\rangle\right| \leq d_{t}(\mathcal{C})-1 \text {. }
$$

(2) This is a consequence of the fact that $d_{t+1} \geq d_{t}+1$ and $d_{k} \leq n$.

(3) This proof is analogous to [22]. The reason for giving it here is to assure oneself that no complications arise from the fact that the weights in $\mathcal{C}^{\perp}$ are measured with respect to the dual poset.

We show that for any $1 \leq s \leq n-k-1$,

$$
n+1-d_{s}\left(\mathrm{C}^{\perp}\right) \notin\left\{d_{r}(\mathcal{C}): 1 \leq r \leq k\right\} .
$$

Let $t=k+s-d_{s}\left(\mathrm{C}^{\perp}\right)$. We consider two cases (one of which can be void), namely, $r \leq t$ and $r \geq t+1$ and show that for each of them, $n+1-d_{s}\left(\mathrm{C}^{\perp}\right) \neq d_{r}(\mathrm{C})$.

Take an $s$-dimensional subcode $\mathcal{D}_{s} \subseteq \mathrm{C}^{\perp}$ such that $\left|\left\langle\operatorname{supp} \mathcal{D}_{s}\right\rangle \overleftarrow{\widehat{p}^{\prime}}\right|=d_{s}\left(\mathrm{C}^{\perp}\right)$. Form a parity-check matrix of the code $\mathcal{C}$ whose first rows are some $s$ linearly independent vectors from $\mathcal{D}_{s}$. Let $D$ be the complement of $\left\langle\operatorname{supp} \mathcal{D}_{s}\right\rangle$ in the set of coordinates. Let the submatrix of $H$ formed of all the columns in $D$ be denoted by $H[D]$. The rank of $H[D]$ is at most $n-k-s$ and its corank (dimension of the null space) is at least

$$
|D|-(n-k-s)=n-d_{s}\left(\mathrm{C}^{\perp}\right)-n+k+s=k+s-d_{s}\left(\mathrm{C}^{\perp}\right) .
$$


Then $d_{t}(\mathrm{C}) \leq|D|=n-d_{s}\left(\mathrm{C}^{\perp}\right)$ and so $d_{r}(\mathrm{C}) \leq n-d_{s}\left(\mathrm{C}^{\perp}\right), 1 \leq r \leq t$.

Now let us show that $d_{t+i}(\mathrm{C}) \neq n+1-d_{s}\left(\mathrm{C}^{\perp}\right)$ for all $1 \leq i \leq k-t$. Assume the contrary and consider a generator matrix $G$ of $\mathcal{C}$ with the first $t+i$ rows corresponding to the subcode $\mathcal{D}_{t+i} \subseteq \mathcal{C}$ with $\left|\left\langle\operatorname{supp} \mathcal{D}_{t+i}\right\rangle_{\overrightarrow{\mathcal{p}}}\right|=d_{t+i}(\mathcal{C})$. Let $D$ be the complement of $\left\langle\operatorname{supp} \mathcal{D}_{t+i}\right\rangle$ in the set of coordinates. Then $G[D]$ is a $k \times(n-$ $\left.d_{t+i}(\mathrm{C})\right)$ matrix of rank $k-t-i$. By part (2) of the lemma, $n-d_{t+i}(\mathcal{C}) \geq k-t-i$, so

$$
\begin{aligned}
\operatorname{dim} \operatorname{ker}(G[D]) & \geq n-d_{t+i}(\mathcal{C})-k+t+i \\
& =s+i-\left(d_{s}\left(\mathfrak{C}^{\perp}\right)+n-d_{t+i}(\mathcal{C})\right) \\
& =s+i-1,
\end{aligned}
$$

where the first equality follows on substituting the value of $k$ and the second one by using the assumption. Hence $d_{s+i-1}\left(\mathrm{C}^{\perp}\right) \leq|D|=d_{s}\left(\mathrm{C}^{\perp}\right)-1$, which contradicts part (1) of the lemma.

(4) Follows by standard linear-algebraic arguments.

Definition 2.3. A linear code $\mathcal{C}[n, k, d]$ is called NMDS if $d(\mathcal{C})=n-k$ and $d_{2}(\mathrm{C})=n-k+2$.

Closely related is the notion of almost-MDS code where we have only the constraint that $d(\mathrm{C})=n-k$ and there is no constraint on $d_{2}(\mathrm{C})$. In this work, we focus only on NMDS codes. The next set of properties of NMDS codes can be readily obtained as generalizations of the corresponding properties of NMDS codes in the Hamming space [6].

Lemma 2.4. Let $\mathcal{C} \subseteq \mathbb{F}_{q}^{n}$ be a linear $[n, k, d]$ code in the poset $\overrightarrow{\mathcal{P}}$.

(1) $\mathcal{C}$ is NMDS if and only if

(a) Any $n-k-1$ l.a. columns of the parity check matrix $H$ are linearly independent.

(b) There exist $n-k$ l.a. linearly dependent columns of $H$.

(c) Any l.a. $n-k+1$ columns of $H$ are full ranked.

(2) If $\mathrm{C}$ is $N M D S$, so is its dual $\mathrm{C}^{\perp}$.

(3) $\mathcal{C}$ is $N M D S$ if and only if $d(\mathcal{C})+d\left(\mathrm{C}^{\perp}\right)=n$.

(4) If $\mathcal{C}$ is $N M D S$ then there exists an NMDS code with parameters $[n-1, k-$ $1, d]$ and an NMDS code with parameters $[n-1, k, d]$.

Proof. (1) Parts (a) and (b) are immediate. Part (c) is obtained from Lemma 2.2.

(2) From Lemma 2.2 we obtain

$$
\left\{n+1-d_{t}\left(\mathcal{C}^{\perp}\right), 1 \leq t \leq n-k\right\}=\{1, \ldots, n-k-1, n-k+1\} .
$$

Hence $d\left(\mathrm{C}^{\perp}\right)=k$ and $d_{2}\left(\mathrm{C}^{\perp}\right)=k+2$.

(3) Let $d(\mathcal{C})+d\left(\mathcal{C}^{\perp}\right)=n$. Then

$$
d_{2}\left(\mathfrak{C}^{\perp}\right) \geq d\left(\mathfrak{C}^{\perp}\right)+1=n-d(\mathfrak{C})+1,
$$

but then by Lemma $2.2(3), d_{2}\left(\mathrm{C}^{\perp}\right) \geq n-d(\mathrm{C})+2$. Next,

$$
n \geq d_{n-k}\left(\mathcal{C}^{\perp}\right) \geq d_{2}\left(\mathcal{C}^{\perp}\right)+n-k-2 \geq 2 n-k-d,
$$

which implies that $d \geq n-k$. This leaves us with the possibilities of $d=n-k$ or $n-k+1$, but the latter would imply that $d(\mathcal{C})+d\left(\mathrm{e}^{\perp}\right)=n+2$, so $d=n-k$. Further, $d_{2}(\mathcal{C}) \geq n-d\left(\mathfrak{C}^{\perp}\right)+2=n-k+2$, as required. The converse is immediate. 
(4) To get a $[n-1, k-1, d]$ NMDS code, delete a column of the parity check matrix $H$ of $\mathcal{C}$ preserving a set of $n-k$ l.a. linearly dependent columns. To get a $[n-1, k, d]$ NMDS code, delete a column of the generator matrix $G$ of $\mathcal{C}$ preserving a set of $k+1$ r.a. columns which contains $k$ r.a. linearly dependent columns.

Lemma 2.5. Let $\mathcal{C}$ be a linear poset code in $\overrightarrow{\mathcal{P}}$ with distance $d$ and let $\mathcal{C}^{\perp}$ be its dual code. Then the matrix $M$ whose rows are the codewords of $\mathcal{C}^{\perp}$ forms an orthogonal array of strength $d-1$ with respect to $\overrightarrow{\mathcal{P}}$.

Proof. Follows because (1), $\mathrm{C}^{\perp}$ is the linear span of the parity-check matrix $H$ of $\mathcal{C}$; and (2), any $d-1$ l.a. columns of $H$ are linearly independent.

\section{NMDS CODES AND DISTRIBUTIONS}

In this section we prove a characterization of NMDS poset codes and then use this result to establish a relationship between NMDS codes in the ordered Hamming space $\mathbb{F}_{q}^{r, n}$ and uniform distributions of points in the unit cube $U^{n}$. In our study of NMDS codes in the following sections, we analyze the properties of the code simultaneously as a linear code and as a linear orthogonal array.

Define the $I$-neighborhood of a poset code $\mathcal{C}$ with respect to an ideal $I$ as

$$
B_{I}(\mathfrak{C})=\bigcup_{c \in \mathcal{C}} B_{I}(c)
$$

where $B_{I}(x)=\left\{v \in \mathbb{F}_{q}^{n}: \operatorname{supp}(v-x) \subseteq I\right\}$. We will say that a linear $k$-dimensional code $\mathcal{C}$ forms an $I$-tiling if there exists a partition $\mathcal{C}=\mathcal{C}_{1} \cup \cdots \cup \mathcal{C}_{q^{k-1}}$ into equal parts such that the $I$-neighborhoods of its parts are disjoint. If in addition the $I$-neighborhoods form a partition of $\mathbb{F}_{q}^{n}$, we say $\mathcal{C}$ forms a perfect I-tiling.

Theorem 3.1. Let $\mathcal{C} \subseteq \mathbb{F}_{q}^{n}$ be an $[n, k, d]$ linear code in the poset $\overrightarrow{\mathcal{P}}$. $\mathcal{C}$ is NMDS if and only if

(1) For any $I \subset \overrightarrow{\mathcal{P}},|I|=n-k+1$, the code $\mathcal{C}$ forms a perfect I-tiling.

(2) There exists an ideal $I \subset \overrightarrow{\mathcal{P}},|I|=n-k$ with respect to which $\mathcal{C}$ forms an I-tiling. No smaller-sized ideals with this property exist.

Proof. Let $\mathcal{C}$ be NMDS and let $I$ be an ideal of size $n-k+1$. Let $H[I]$ be the submatrix of the parity-check matrix $H$ of $\mathcal{C}$ obtained from $H$ by deleting all the columns not in $I$. Since $\operatorname{rk}(H[I])=n-k$, the space $\operatorname{ker}(H[I])$ is one-dimensional. Let $\mathcal{C}_{1}=\operatorname{ker}(H(I))$ and let $\mathcal{C}_{j}$ be the $j$ th coset of $\mathcal{C}_{1}$ in $\mathcal{C}, j=2, \ldots, q^{k-1}$. By Lemma 2.5 the code $\mathcal{C}$ forms an orthogonal array of strength $k-1$ and index $q$ in $\overleftarrow{\mathcal{P}}$. Therefore, every vector $z \in \mathbb{F}_{q}^{k-1}$ appears exactly $q$ times in the restrictions of the codevectors $c \in \mathcal{C}$ to the coordinates of $J=I^{c}$. Thus, $c^{\prime}[J]=c^{\prime \prime}[J]$ for any two vectors $c^{\prime}, c^{\prime \prime} \in \mathcal{C}_{i}, i=1, \ldots, q^{k-1}$ and $c^{\prime}[J] \neq c^{\prime \prime}[J] c^{\prime} \in \mathcal{C}_{i}, c^{\prime \prime} \in \mathcal{C}_{j}, 1 \leq i<$ $j \leq q^{k-1}$. This implies that $\mathcal{C}$ forms a perfect $I$-tiling, which proves assumption 1 of the theorem. To prove assumption 2, repeat the above argument taking $I$ to be the support of a minimum-weight codeword in $\mathrm{C}$.

To prove the converse, let $I \subseteq \overrightarrow{\mathcal{P}},|I|=n-k+1$ be an ideal and let $\mathcal{C}_{1}, \ldots, \mathcal{C}_{q^{k-1}}$ be a partition of $\mathcal{C}$ with $\left|\mathcal{C}_{i}\right|=q$ for all $i$, that forms a perfect $I$-tiling. This implies that $c^{\prime}\left[I^{c}\right] \neq c^{\prime \prime}\left[I^{c}\right], c^{\prime} \in \mathcal{C}_{i}, c^{\prime \prime} \in \mathcal{C}_{j}, 1 \leq i<j \leq q^{k-1}$. In other words, $\mathcal{C}$ forms an orthogonal array with respect to $\overleftarrow{\mathcal{P}}$ of index $q$ and strength $k-1$. We conclude that 
$d\left(\mathrm{C}^{\perp}\right)=k$ or $k+1$. If it is the latter, then $\mathcal{C}^{\perp}$ is MDS with respect to $\overleftarrow{\mathcal{P}}$ and so is $\mathcal{C}$ with respect to $\overrightarrow{\mathcal{P}}$, in violation of assumption 2 . So $d\left(\mathrm{C}^{\perp}\right)=k$ and $d(\mathcal{C}) \leq n-k$. If the inequality is strict, there exists an ideal $I$ of size $<n-k$ that supports a onedimensional subcode of $\mathcal{C}$. Then $\mathcal{C}$ forms an $I$-tiling which contradicts assumption 2 .

It remains to prove that $d_{2}(\mathcal{C})=n-k+2$. Assume the contrary, i.e., that there exists a 2-dimensional subcode $\mathcal{B} \subset \mathcal{C}$ whose l.a. support forms an ideal $I \subset \overrightarrow{\mathcal{P}}$ of size $n-k+1$. The $q^{2}$ vectors of $\mathcal{B}$ all have zeros in $I^{c}$ which contradicts the fact that $\mathcal{C}$ forms an orthogonal array of index $q$.

Next, we use this characterization to relate codes in the ordered Hamming space $\mathbb{F}_{q}^{r, n}$ to distributions. An idealized uniformly distributed point set $\mathcal{C}$ would satisfy the property that for any measurable subset $A \subset U^{n}$,

$$
\frac{1}{|\mathcal{C}|} \sum_{x \in \mathcal{C}} 1(x \in A)=\operatorname{vol}(A) \text {. }
$$

Distributions that we consider, and in particular $(t, m, n)$-nets, approximate this property by restricting the subsets $A$ to be boxes with sides parallel to the coordinate axes.

Let

$$
\mathcal{E} \triangleq\left\{E=\prod_{i=1}^{n}\left[\frac{a_{i}}{q^{d_{i}}}, \frac{a_{i}+1}{q^{d_{i}}}\right): 0 \leq a_{i}<q^{d_{i}}, 0 \leq d_{i} \leq r, 1 \leq i \leq n\right\}
$$

be a collection of elementary intervals in the unit cube $U^{n}=[0,1)^{n}$. An arbitrary collection of $q^{k}$ points in $U^{n}$ is called an $[n r, k]$ distribution in the base $q$ (with respect to $\mathcal{E}$ ). A distribution is called optimal if every elementary interval of volume $q^{-k}$ contains exactly one point [20]. A related notion of $(t, m, n)$-nets, introduced by Niederreiter [16], is obtained if we remove the upper bound on $l_{i}$ (i.e., allow that $\left.0 \leq l_{i}<\infty\right)$ and require that every elementary interval of volume $q^{t-m}$ contain exactly $q^{t}$ points.

An ordered code gives rise to a distribution of points in the unit cube via the following procedure. A codevector $\left(c_{11}, \ldots, c_{1 r}, \ldots, c_{n 1}, \ldots, c_{n r}\right) \in \mathbb{F}_{q}^{r, n}$ is mapped to $x=\left(x_{1}, \ldots, x_{n}\right) \in U^{n}$ by letting

$$
x_{i}=\sum_{j=1}^{r} c_{i j} q^{j-r-1}, 1 \leq i \leq n .
$$

In particular, an $(m-t, n, r, q)$ OOA of index $q^{t}$ and size $q^{m}$ corresponds to a distribution in which every elementary interval of volume $q^{t-m}$ contains exactly $q^{t}$ points, and an $(m-t, n, m-t, q)$ OOA of index $q^{t}$ and size $q^{m}$ gives rise to a $(t, m, n)$-net $[12,15]$.

Proposition 3.2. (Skriganov [20]) An $[n r, k, d] M D S$ code in the ordered metric exists if and only if there exists an optimal $[n r, k]$ distribution.

Skriganov [21] also considers the concept of nearly-MDS codes whose distance asymptotically tends to the distance of MDS codes, and shows how these codes can give rise to distributions.

The next theorem whose proof is immediate from Theorem 3.1 relates ordered NMDS codes and distributions. 
Theorem 3.3. Let $\mathcal{C}$ be a linear $[n r, k, d]$ code in $\mathbb{F}_{q}^{r, n}$ and let $P(\mathcal{C})$ be the corresponding set of points in $U^{n}$. Then $\mathcal{C}$ is NMDS if and only if

(1) Any elementary interval of volume $q^{-(k-1)}$ has exactly $q$ points of $P(\mathfrak{C})$.

(2) There exists an elementary interval $\prod_{i=1}^{n}\left[0, q^{-l_{i}}\right)$ of volume $q^{-k}$ containing exactly $q$ points and no smaller elementary intervals of this form containing exactly $q$ points exist.

Corollary 3.4. An $[n r, k, d] N M D S$ code $\mathcal{C}$ in the ordered Hamming space forms a $(k-1, n, r, q)$ OOA of index $q$. The corresponding distribution $P(\mathcal{C}) \subset U^{n}$ forms a $(k-r, k, n)$-net for $k-1 \geq r$.

Remark 3.5. Distributions of points in the unit cube obtained from NMDS codes have properties similar to those of distributions obtained from MDS codes. In particular, the points obtained from an $[n r, k, d]$ MDS code in $\mathbb{F}_{q}^{r, n}$ satisfy part (1) of Theorem 3.3 and give rise to a $(k-r, k, n)$-net for $k \geq r$ [20].

\section{Weight distribution of NMDS CODES}

Let $\Omega(I)$ be the set of maximal elements of an ideal $I$ and let $\tilde{I} \triangleq I \backslash \Omega(I)$.

Let $\mathcal{C}$ be an NMDS $[n, k, d]$ linear poset code. Let $A_{I} \triangleq\{x \in \mathcal{C}:\langle\operatorname{supp} x\rangle=I\}$ be the number of codewords with l.a. support exactly $I$ and let $A_{s}=\sum_{I:|I|=s} A_{I}$.

Theorem 4.1. The weight distribution of $\mathcal{C}$ has the following form:

$$
\begin{array}{r}
A_{s}=\sum_{I \in \mathcal{J}_{s}} \sum_{l=0}^{s-d-1}(-1)^{l}\left(\begin{array}{c}
|\Omega(I)| \\
l
\end{array}\right)\left(q^{s-d-l}-1\right)+(-1)^{s-d} \sum_{I \in \mathcal{J}_{s}} \sum_{J \in \mathcal{J}_{d}(I), J \supseteq \tilde{I}} A_{J}, \\
n \geq s \geq d,
\end{array}
$$

where $\mathcal{J}_{s} \triangleq\{I \subseteq \overrightarrow{\mathcal{P}}:|I|=s\}$ and $\mathcal{J}_{s}(I) \triangleq\{J: J \subseteq I,|J|=s\}$.

Proof. The computation below is driven by the fact that ideals are fixed by the sets of their maximal elements. Additionally, we use the fact that any $k-1$ r.a. coordinates of the code $\mathcal{C}$ support an orthogonal array of strength $k-1$.

The number of codewords of weight $s$ is given by $A_{s}=\left|\cup_{I \in \mathcal{J}_{s}} \mathrm{C} \cap S_{I}\right|$, where $S_{I} \triangleq\left\{x \in \mathbb{F}_{q}^{n}:\langle\operatorname{supp} x\rangle=I\right\}$ is the sphere with l.a. support exactly $I$. The above expression can be written as

$$
\left|\bigcup_{I \in \mathcal{J}_{s}} \mathfrak{e} \cap S_{I}\right|=\sum_{I \in \mathcal{J}_{s}}\left(\left|\mathcal{e} \cap B_{I}^{*}\right|-\left|\bigcup_{J \in \mathcal{J}_{s-1}(I)} \mathcal{C} \cap B_{J}^{*}\right|\right),
$$

where $B_{I} \triangleq\left\{x \in \mathbb{F}_{q}^{n}:\langle\operatorname{supp} x\rangle_{\vec{p}} \subseteq I\right\}$ and $B_{I}^{*} \triangleq B_{I} \backslash \mathbf{0}$. We determine the cardinality of the last term using the inclusion-exclusion principle.

$$
\begin{aligned}
\left|\bigcup_{J \in \mathcal{J}_{s-1}(I)} \mathcal{C} \cap B_{J}^{*}\right|= & \sum_{J \in \mathcal{J}_{s-1}(I)}\left|\mathcal{C} \cap B_{J}^{*}\right|-\sum_{J_{1} \neq J_{2} \in \mathcal{J}_{s-1}(I)}\left|\mathcal{C} \cap B_{J_{1}}^{*} \cap B_{J_{2}}^{*}\right|+\cdots \\
& +(-1)^{|\Omega(I)|-1} \sum_{J_{1} \neq \cdots \neq J_{|\Omega(I)|} \in \mathcal{J}_{s-1}(I)}\left|\mathcal{e} \cap\left(\bigcap_{i} B_{J_{i}}^{*}\right)\right| .
\end{aligned}
$$

Since $\mathcal{C}^{\perp}$ has minimum distance $k$, $\mathcal{C}$ forms an orthogonal array of strength $k-1$ with respect to the dual poset $\overleftarrow{\mathcal{P}}$. This provides us with an estimate for each individual term in (4.2) as described below. For distinct $J_{1}, \ldots, J_{l} \in \mathcal{J}_{s-1}(I)$, we 
let $J \triangleq \cap_{i=1}^{l} J_{i}$. Using the fact that $J$ does not contain $l$ maximal elements of $I$, we get

$$
\mid\left\{\left\{J_{1}, \ldots, J_{l}\right\}: J_{i} \text { distinct, } J_{i} \in \mathcal{J}_{s-1}(I), i=1, \ldots, l\right\} \mid=\left(\begin{array}{c}
|\Omega(I)| \\
l
\end{array}\right) .
$$

For any $s \geq d+1$ consider the complement $I^{c}$ of an ideal $I \in \mathcal{J}_{s}$. Since $\left|I^{c}\right| \leq$ $n-d-1=k-1$, the code $\mathcal{C}$ supports an orthogonal array of strength $n-s$ and index $q^{s-d}$ in the coordinates defined by $I^{c}$. Since $\cap_{i=1}^{l} B_{J_{i}}^{*}=B_{J}^{*}$ and since $B_{J}^{*}$ does not contain the $\mathbf{0}$ vector, we obtain

$$
\left|\mathcal{e} \cap\left(\bigcap_{i=1}^{l} B_{J_{i}}^{*}\right)\right|=q^{s-d-l}-1, \quad 1 \leq l \leq s-d-1 .
$$

Finally, for $l=s-d$ we obtain $\left|\mathcal{C} \cap\left(\cap_{i=1}^{l} B_{J_{i}}^{*}\right)\right|=A_{J}$, and

$$
\left|\bigcup_{J \in \mathcal{J}_{s-1}(I)} \mathcal{C} \cap B_{J}^{*}\right|=\sum_{l=1}^{s-d-1}(-1)^{l-1}\left(\begin{array}{c}
|\Omega(I)| \\
l
\end{array}\right)\left(q^{s-d-l}-1\right)+\sum_{J \in \mathcal{J}_{d}(I), J \supseteq \tilde{I}}(-1)^{s-d-1} A_{J},
$$

which implies

$$
\begin{aligned}
\sum_{I \in \mathcal{J}_{s}}\left|\mathcal{e} \cap S_{I}\right|=\sum_{I \in \mathcal{J}_{s}}\left(\left(q^{s-d}-1\right)-\left(\sum_{l=1}^{s-d-1}(-1)^{l-1}\left(\begin{array}{c}
|\Omega(I)| \\
l
\end{array}\right)\left(q^{s-d-l}-1\right)\right.\right. \\
\left.\left.+\sum_{J \in \mathcal{J}_{d}(I), J \supseteq \tilde{I}}(-1)^{s-d-1} A_{J}\right)\right) .
\end{aligned}
$$

As a corollary of the above theorem, we obtain the weight distribution of NMDS codes in the ordered Hamming space $\mathbb{F}_{q}^{r, n}$. By definition, the number of vectors of ordered weight $s$ in a code $\mathcal{C} \in \mathbb{F}_{q}^{r, n}$ equals $A_{s}=\sum_{e:|e|^{\prime}=s} A_{e}$, where $A_{e}$ is the number of codevectors of shape $e$.

Corollary 4.2. The weight distribution of an ordered $N M D S$ code $\mathcal{C} \in \mathbb{F}_{q}^{r, n}$ is given by

$$
\begin{aligned}
A_{s}=\sum_{l=0}^{s-d-1}(-1)^{l}\left(\sum_{e:|e|^{\prime}=s}\left(\begin{array}{c}
|e| \\
l
\end{array}\right)\left(\begin{array}{c}
n \\
e_{0}, \ldots, e_{r}
\end{array}\right)\right)\left(q^{s-d-l}-1\right)+ & \\
& +(-1)^{s-d} \sum_{e:|e|^{\prime}=d} N_{s}(e) A_{e}, \quad s=d, d+1, \ldots, n,
\end{aligned}
$$

where

$$
N_{s}(e) \triangleq \sum_{f:|f|^{\prime}=s}\left(\begin{array}{c}
e_{r-1} \\
f_{r}-e_{r}
\end{array}\right)\left(\begin{array}{c}
e_{r-2} \\
\left(f_{r}+f_{r-1}\right)-\left(e_{r}+e_{r-1}\right)
\end{array}\right) \cdots\left(\begin{array}{c}
e_{0} \\
|f|-|e|
\end{array}\right) .
$$

Proof. Recall that the shape of an ideal $I$ is shape $(I)=e=\left(e_{1}, \ldots, e_{r}\right)$, where $e_{j}, j=1, \ldots, r$ is the number of chains of length $j$ contained in $I$. We obtain $|\Omega(I)|=|e|$ and

$$
\sum_{I \in \mathcal{J}_{s}}\left(\begin{array}{c}
|\Omega(I)| \\
l
\end{array}\right)=\sum_{e:|e|^{\prime}=s}\left(\begin{array}{c}
|e| \\
l
\end{array}\right)\left(\begin{array}{c}
n \\
e_{0}, \ldots, e_{r}
\end{array}\right)
$$




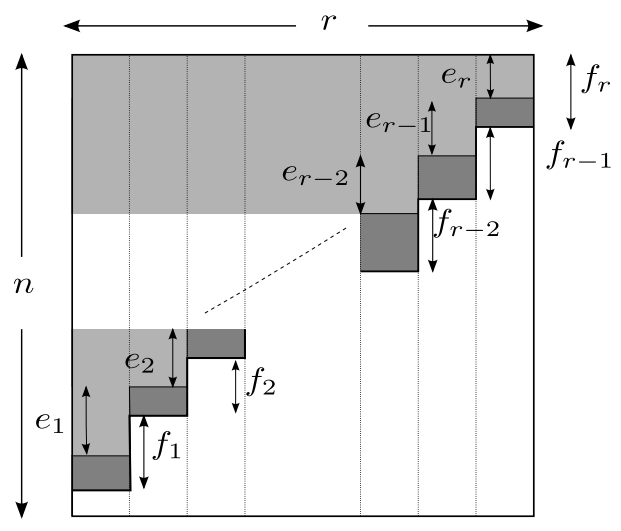

Light gray region: ideal $J$ of shape $e$.

Light gray + Dark gray region: ideal $I$ of shape $f$.

Figure 1. To the proof of Corollary 4.2

To determine the last term in (4.1), we rewrite it as

$$
\begin{aligned}
\sum_{I \in \mathcal{J}_{s}} \sum_{J \in \mathcal{J}_{d}(I), J \supseteq \tilde{I}} A_{J} & =\sum_{J \in \mathcal{J}_{d}}\left|\left\{I \in \mathcal{J}_{s}: \tilde{I} \subseteq J \subseteq I\right\}\right| A_{J} \\
& =\sum_{e:|e|^{\prime}=d} N_{s}(e) \sum_{J: \operatorname{shape}(J)=e} A_{J},
\end{aligned}
$$

where $N_{s}(e)=\mid\left\{I \in \mathcal{J}_{s}: \tilde{I} \subseteq J \subseteq I, J\right.$ fixed, shape $\left.(J)=e\right\} \mid$.

Clearly, $\sum_{J \text { :shape }(J)=e} A_{J}=A_{e}$, and so we only need to determine the quantity $N_{s}(e)$ in the above summation. Let $J$ be an ideal as shown in Fig. 1. The ideals $I$ which satisfy the constraints in the set defined by $N_{s}(e)$ have the form as shown in Fig. 1. Letting $f=\operatorname{shape}(I)$, we note that the components of the shape $f$ must satisfy

$$
\begin{aligned}
f_{r} & \geq e_{r}, \\
f_{r}+f_{r-1} & \geq e_{r}+e_{r-1} \geq f_{r}, \\
\vdots & \\
f_{1}+\cdots+f_{r}=|f| & \geq|e|=e_{1}+\cdots+e_{r} \geq f_{2}+\cdots+f_{s}, \\
\text { and }|f|^{\prime} & =s .
\end{aligned}
$$

It is now readily seen that the cardinality of the set

$$
\left\{I \in \mathcal{J}_{s}: \tilde{I} \subseteq J \subseteq I, J \text { fixed, } \operatorname{shape}(J)=e\right\}
$$

is given by the formula for $N_{s}(e)$ as described in (4.3).

Remark: For $r=1$ we obtain $|e|=|e|^{\prime}=e_{1}=d,|f|=f_{1}=s$ and $N_{s}(e)=$ $\left(\begin{array}{l}n-d \\ s-d\end{array}\right)$. Thus we recover the expression for the weight distribution of an NMDS code in Hamming space [6]:

$$
A_{s}=\sum_{l=0}^{s-d-1}(-1)^{l}\left(\begin{array}{l}
s \\
l
\end{array}\right)\left(\begin{array}{l}
n \\
s
\end{array}\right)\left(q^{s-d-l}-1\right)+(-1)^{s-d}\left(\begin{array}{c}
n-d \\
s-d
\end{array}\right) A_{d} .
$$


Unlike the case of poset MDS codes [11], the weight distribution of NMDS codes is not completely known until we know the number of codewords with l.a. support $J$ for every ideal of weight $J$ of size $d$. In particular, for NMDS codes in the ordered Hamming space we need to know the number of codewords of every shape $e$ with $|e|^{\prime}=d$. This highlights the fact that the combinatorics of codes in the poset space (ordered space) is driven by ideals (shapes) and their support sizes, and that the weight distribution is a derivative invariant of those more fundamental quantities.

As a final remark we observe that, given that $d(\mathcal{C})=n-k$, the assumption $d\left(\mathrm{C}^{\perp}\right)=k$ (or the equivalent assumption $d_{2}(\mathrm{C})=n-k+2$ ) ensures that the only unknown components of the weight distribution of $\mathcal{C}$ correspond to ideals of size $d$. If instead we consider a code of defect $s$, i.e., a code with $d(\mathcal{C})=(n-k+1)-s, s \geq 2$, it will be possible to compute its weight distribution using the components $A_{J}, d \leq$ $|J| \leq n-d\left(\mathrm{C}^{\perp}\right)$ (provided that we know $\left.d\left(\mathrm{C}^{\perp}\right)\right)$. In the case of the Hamming metric this was established in [8].

\section{Constructions of NMDS codes}

In this section we present some simple constructions of NMDS codes in the ordered Hamming space for the cases $n=1,2,3$. We are not aware of any general code family of NMDS codes for larger $n$.

$\mathbf{n}=\mathbf{1}$ : For $n=1$ the construction is quite immediate once we recognize that an NMDS $[r, k, d]$ code is also an OOA of r.a. strength $k-1$ and index $q$. Let $I_{l}$ denote the identity matrix of size $l$. Let $x=\left(x_{1}, \ldots, x_{r}\right)$ be any vector of 1 .a. weight $d=r-k$, i.e. $x_{d} \neq 0$ and $x_{l}=0, l=d+1, \ldots, r$. Then the following matrix of size $k \times r$ generates an NMDS code with the above parameters

$$
\left[\begin{array}{ccc}
x_{1} \ldots x_{d} & 0 & \mathbf{0} \\
M & \mathbf{0} & I_{k-1}
\end{array}\right]
$$

where the 0 s are zero vectors (matrices) of appropriate dimensions and $M \in$ $\mathbb{F}_{q}^{(k-1) \times d}$ is any arbitrary matrix.

$\mathbf{n = 2}$ : Let $D_{l}=\left[\begin{array}{ccc}0 & \ldots & 1 \\ \vdots & \ddots & \ddots \\ 1 & \ldots & \vdots \\ 1 & \ldots & 0\end{array}\right]$ be the $l \times l$ matrix with 1 along the inverse diagonal and 0 elsewhere. Let $u$ and $v$ be two vectors of length $r$ in $\mathbb{F}_{q}^{r, 1}$ and l.a. weights $r-k_{1}$ and $r-k_{2}$ respectively and let $K=k_{1}+k_{2}$. The following matrix generates a $[2 r, K, 2 r-K]$ linear NMDS code in $\mathbb{F}_{q}^{r, 2}$,

$$
\left[\begin{array}{cccc|cccc}
u_{1} \ldots u_{r-k_{1}-1} & u_{r-k_{1}} & 0 & \mathbf{0} & v_{1} \ldots v_{r-k_{2}-1} & v_{r-k_{2}} & 0 & \mathbf{0} \\
\mathbf{0} & 0 & 1 & 0 & \mathbf{0} & 0 & 1 & 0 \\
\mathbf{0} & \mathbf{0} & \mathbf{0} & I_{k_{1}-1} & E_{r}\left(k_{1}, k_{2}\right) & \mathbf{0} & \mathbf{0} & \mathbf{0} \\
E_{r}\left(k_{2}, k_{1}\right) & \mathbf{0} & \mathbf{0} & \mathbf{0} & \mathbf{0} & \mathbf{0} & \mathbf{0} & I_{k_{2}-1}
\end{array}\right],
$$

where $E_{r}(i, j)$ is an $(i-1) \times(r-j-1)$ matrix which has the following form:

$$
E_{r}(i, j)= \begin{cases}{\left[\frac{D_{r-j-1}}{\mathbf{0}_{(i+j-r) \times(r-j-1)}}\right],} & i+j>r, \\ {\left[\mathbf{0}_{(i-1) \times(r-i-j)} \mid D_{i-1}\right],} & i+j \leq r .\end{cases}
$$

From the form of the generator matrix it can be seen that any $K-1$ r.a. columns of the above matrix are linearly independent. But the last $k_{1}$ and $k_{2}$ columns from the first and the second blocks respectively are linearly dependent. This implies 
that it forms an OOA of r.a. strength exactly $K-1$. Hence the dual of the code has distance $K$. Finally, the minimum weight of any vector produced by this generator matrix is $2 r-K$. Hence by Lemma 2.4, this matrix generates an NMDS code.

$\mathbf{n}=3$ : $\quad$ For $n=3$, we have an NMDS code with very specific parameters. Let $u, v, w \in \mathbb{F}_{q}^{r, 1}$ be three vectors of l.a. weight $r-2$ each. Then the matrix shown below is the generator matrix of a $[3 r, 6, d]$ code in base $q \geq 3$. It is formed of three blocks, corresponding to the three dimensions given by $n$. Here $\mathbf{0}$ is a $1 \times(r-6)$ zero vector.

$\left[\begin{array}{ccccccc}u_{1} \ldots u_{r-6} & u_{r-5} & u_{r-4} & u_{r-3} & u_{r-2} & 0 & 0 \\ \mathbf{0} & 0 & 0 & 0 & 0 & 1 & 0 \\ \mathbf{0} & 0 & 1 & 0 & 0 & 1 & 0 \\ \mathbf{0} & 1 & 0 & 0 & 0 & 0 & 1 \\ \mathbf{0} & 0 & 1 & 0 & 0 & 0 & 1 \\ \mathbf{0} & 0 & 0 & 1 & 0 & 0 & 0\end{array}\right]$
$\left[\begin{array}{ccccccc}v_{1} \ldots v_{r-6} & v_{r-5} & v_{r-4} & v_{r-3} & v_{r-2} & 0 & 0 \\ \mathbf{0} & 0 & 0 & 0 & 0 & 1 & 0 \\ \mathbf{0} & 0 & 1 & 0 & 0 & 0 & 0 \\ \mathbf{0} & 0 & 0 & 1 & 0 & 0 & 0 \\ \mathbf{0} & 0 & 1 & 0 & 0 & 0 & 1 \\ \mathbf{0} & 1 & 0 & 0 & 0 & 0 & 1\end{array}\right]$
$\left[\begin{array}{ccccccc}w_{1} \ldots w_{r-6} & w_{r-5} & w_{r-4} & w_{r-3} & w_{r-2} & 0 & 0 \\ \mathbf{0} & 0 & 1 & 0 & 0 & 0 & 0 \\ \mathbf{0} & 0 & 0 & 0 & 0 & 1 & 0 \\ \mathbf{0} & 0 & 1 & 0 & 0 & 0 & 1 \\ \mathbf{0} & 0 & 0 & 1 & 0 & 0 & 0 \\ \mathbf{0} & 1 & 0 & 0 & 0 & 0 & 1\end{array}\right]$.

\section{REFERENCES}

[1] T. L. Alderson, A. A. Bruen, and R. Silverman, Maximum distance separable codes and arcs in projective spaces, J. Combin. Theory Ser. A 114 (2007), no. 6, 1101-1117.

[2] A. Barg and P. Purkayastha, Bounds on ordered codes and orthogonal arrays, Moscow Mathematical Journal 9 (2009), no. 2, 211-243.

[3] J. Bierbrauer, A direct approach to linear programming bounds for codes and tms-nets, Des. Codes Cryptogr. 42 (2007), 127-143.

[4] R. A. Brualdi, J.S. Graves, and K. M. Lawrence, Codes with a poset metric, Discrete Math. 147 (1995), no. 1-3, 57-72.

[5] M. de Boer, Almost MDS codes, Des. Codes Cryptogr. 9 (1996), 143-155.

[6] S. Dodunekov and I. Landgev, Near-MDS codes, J. of Geometry 54 (1995), no. 1, 30-43.

[7] S. T. Dougherty and M. M. Skriganov, Maximum distance separable codes in the $\rho$ metric over arbitrary alphabets, Journal of Algebraic Combinatorics 16 (2002), 71-81.

[8] A. Faldum and W. Willems, A characterization of MMD codes, IEEE Trans. Inform. Theory 44 (1998), no. 4, 1555-1558.

[9] A. Ganesan and P. O. Vontobel, On the existence of universally decodable matrices, IEEE Trans. Inform. Theory 53 (2007), no. 7, 2572-2575.

[10] G. G. Günther, Finite field Fourier transform for vectors of arbitrary length, Communications and Cryptography: Two Sides of One Tapestry (R. E. Blahut, Jr. D. J. Costello, U. Maurer, and T. Mittelholzer, eds.), Norwell, MA, and Dordrecht, NL: Kluwer Academic, 1994, pp. 141-153.

[11] J. Y. Hyun and H. K. Kim, Maximum distance separable poset codes, Des. Codes Cryptogr. 28 (2008), no. 3, 247-261. 
[12] K. M. Lawrence, A combinatorial characterization of $(t, m, s)$-nets in base b, J. Combin. Designs 4 (1996), 275-293.

[13] W. J. Martin and D. R. Stinson, Association schemes for ordered orthogonal arrays and (T, M, S)-nets, Canad. J. Math. 51 (1999), no. 2, 326-346.

[14] J. L. Massey and S. Serconek, Linear complexity of periodic sequences: a general theory, Advances in cryptology - CRYPTO '96 (Santa Barbara, CA), Lecture Notes in Comput. Sci., vol. 1109, Springer, Berlin, 1996, pp. 358-371.

[15] G. L. Mullen and W. Ch. Schmid, An equivalence between $(t, m, s)$-nets and strongly orthogonal hypercubes, Journal of Combin. Theory, Ser. A 76 (1996), 164-174.

[16] H. Niederreiter, Low-discrepancy point sets, Monatsh. Math. 102 (1986), no. 2, 155-167.

[17] R. R. Nielsen, A class of Sudan-decodable codes, IEEE Trans. Inform. Theory 46 (2000), no. $4,1564-1572$

[18] M. Yu. Rosenbloom and M. A. Tsfasman, Codes for the m-metric, Problems of Information Transmission 33 (1997), no. 1, 45-52.

[19] R. Roth, Introduction to coding theory, Cambridge University Press, Cambridge, 2006.

[20] M. M. Skriganov, Coding theory and uniform distributions, Algebra i Analiz 13 (2001), no. 2, 191-239, English translation in St. Petersburg Math. J. vol. 13 (2002), no. 2, 301-337.

[21] - On linear codes with large weights simultaneously for the Rosenbloom-Tsfasman and Hamming metrics, J. Complexity 23 (2007), no. 4-6, 926-936.

[22] V. Wei, Generalized Hamming weights for linear codes, IEEE Trans. Inform. Theory 37 (1991), no. 5, 1412-1418.

Department of ECE/Institute for Systems Research, University of Maryland, College Park, MD 20817 and Dobrushin Mathematical Lab., Institute for Problems of Information Transmission, Moscow, Russia

E-mail address: abarg@umd.edu

Department of ECE/Institute for Systems Research, University of Maryland, ColLEGE PARK, MD 20817

E-mail address: ppurka@umd.edu 